\title{
Protective effects of salidroside on chronic heart failure in rats and the underlying mechanisms
}

\author{
Chengxi Zhang ${ }^{1, *, \#, ~ S i n i a n ~ P a n ~}{ }^{2, \#}$, Leile Tang${ }^{1}$, Yesheng Ling ${ }^{1}$, Xiaojing Zhou ${ }^{3}$, Wei Feng ${ }^{3}$ \\ ${ }^{1}$ Department of Cardiology, the Third Affiliated Hospital, Sun Yat-Sen University, Guangzhou, China, ${ }^{2}$ Department of \\ Pediatrics, the Third Affiliated Hospital, Sun Yat-Sen University, Guangzhou, China, ${ }^{3}$ Department of Cardiology, the First \\ People's Hospital of Xinjiang Kashi Area, Kashi, China
}

\begin{abstract}
The present study aimed to investigate the protective effects of salidroside on chronic heart failure (CHF) in rats and to explore the underlying mechanisms. One hundred SD rats were randomly divided into sham-operated, model, and low-, medium- and high-dose salidroside groups. The CHF model was established in later 4 groups. The later 3 groups were intragastrically administrated with 6, 12 and $24 \mathrm{mg} / \mathrm{kg}$ salidroside, respectively, once a day, for continuous 4 weeks. Finally, the serum levels of brain natriuretic peptide (BNP) and interleukin 6 (IL-6), cardiac function indexes, and expression levels of myocardial cysteinyl aspartate-specific proteinase (Caspase)-3, Caspase-9, matrix metalloproteinase-1 (MMP-1) and tissue inhibitor of metalloproteinase-1 (TIMP-1) protein were determined. Results showed that, after treatment, compared with model group, in high-dose salidroside group the heart function indexes were significantly improved $(\mathrm{P}<0.05)$, the serum levels of BNP and IL-6 were significantly decreased $(\mathrm{P}<0.05)$, the expression levels of myocardial Caspase-3, Caspase-9 and MMP-1 protein were significantly decreased $(\mathrm{P}<0.05)$, and the expression level of TIMP-1 protein was significantly increased $(\mathrm{P}<0.05)$. In conclusion, salidroside has obvious protective effects on CHF in rats. The mechanisms may be related to its regulation of cardiomyocyte apoptosis and ventricular remodelingregulation related protein expressions.
\end{abstract}

Keywords: Salidroside/protective effects. Chronic heart failure.

\section{INTRODUCTION}

Chronic heart failure (CHF) is a pathophysiological syndrome characterized by pulmonary circulation and/ or systemic circulation congestion due to heart pumping dysfunction, decreased cardiac output or decreased tissue perfusion in the case of normal venous return (Al-Mohammad, Mant, 2011). The CHF patients often present myocardial remodeling, myocardial cell structure change, or increased myocardial cell apoptosis (Distefano, Sciacca, 2012). CHF is a common outcome of other endstage systemic diseases. With the deepening of population aging and rapid increasing of primary diseases such as hypertension, coronary heart disease, diabetes and hyperlipidemia, the incidence of CHF is obviously rising (Cleland et al., 2016). At present, the clinical treatment

\footnotetext{
*Correspondence: C. Zhang. Department of Cardiology, The Third Affiliated Hospital, Sun Yat-Sen University, 600, Tianhe Road, Tianhe District, Guangzhou 510630, China. Tel.: +86-20-85252168. E-mail: zhangchengxi1@yeah.net ${ }^{\#}$ Contributed equally.
}

of CHF is mainly based on use of drugs. The traditional positive inotropic drugs exert the therapeutic effect through improving the hemodynamics, but the long-term use of these drugs will increase the mortality of patients. China has rich resources in natural medicines. The active substances of many natural medicines have the effects of improving heart function and resisting myocardial ischemia (Yin et al., 2008; Yin et al., 2009). Rhodiola rosea $L$. is the perennial herbaceous plant, which mostly grows in the alpine region with altitude of $2-5 \mathrm{~km}$. Rhodiola rosea $L$. contents unique biological active ingredients including salidroside, tyrosol, rosavin, polysaccharide, flavonoids and saponins. Salidroside is one of the main effective components of Rhodiola rosea $L$.. It has the pharmacological effects in resisting hypoxia, promoting hematopoietic function, improving microcirculation, inhibiting oxidative stress and regulating immune (Zhang et al., 2009; Zhang et al., 2013). In clinic, salidroside is widely used in prevention and treatment of coronary heart disease, hypertension, cerebrovascular disease, climacteric 
syndrome and other diseases (Han et al., 2015). At present, the effect of salidroside on CHF is less reported. It has been reported that, the cysteinyl aspartate-specific proteinase (Caspase)-3, Caspase-9, matrix metalloproteinase-1 (MMP-1) and tissue inhibitor of metalloproteinase-1 (TIMP-1) are involved in the course of heart diseases ( $\mathrm{Su}$ et al., 2003; Sakata et al., 2004; Balakumar, Singh, 2006; Picard et al., 2006). This study established the rat CHF model, investigated the protective effects of salidroside on CHF in rats, and explored the mechanisms related to myocardial Caspase-3, Caspase-9, MMP-1 and TIMP1 expressions. The objective was to provide a basis for application of salidroside to clinical treatment of CHF.

\section{MATERIAL AND METHODS}

\section{Animals and treatment}

One hundred male Sprague Dawley rats ( 8 weeks of age; 180-200 g; Guangdong Experimental Animal Center, Guangzhou, China) were single-cage raised under the condition avoiding strong light and noise (12/12-h day-night cycle; free to feed and water). The rats were randomly divided into sham-operated, model, and low-, medium- and high-dose salidroside groups, 20 rats in each group. The CHF model was established in later 4 groups. The rats in low-, medium- and high-dose salidroside groups were intragastrically administrated with salidroside (purity $\geq 98 \%$; Beijing Suolaibao Technology Co. Ltd., Beijing, China), with dose of 6, 12 and $24 \mathrm{mg} /$ $\mathrm{kg}$, respectively. The sham-operated and model groups were intragastrically administrated with equal volume of normal saline. The administration was performed once per day, for continuous 4 weeks.

\section{Establishment of CHF model in rats}

CHF model in rats was established using the coronary artery ligation method. After two week of adaptive feeding, the rats were anaesthetized by intraperitoneal injection of chloral hydrate with dose of $100 \mathrm{mg} / \mathrm{kg}$, followed by fixation in supine position and skin preserving on chest. The electrocardiogram (ECG) electrode was subcutaneously embedded in the right upper limb and left lower limb, and the normal lead II ECG was recorded. The left fourth-fifth intercostal chest skin was cut open, and the pectoral was bluntly separated. The chest was opened using forceps, and the heart was exposed. The right chest was gently pressed, and the heart was gently squeezed out of the thoracic cavity. The threading was performed at the position $3 \mathrm{~mm}$ from the initial section of left anterior descending coronary artery between the pulmonary artery cone and left atrial appendage. Then, the heart was placed to the thoracic cavity. After squeezing out the air, the thoracic cavity was closed, and the spontaneous breathing was restored. After stabilization for $10 \mathrm{~min}$, the coronary artery was ligated. The ECG changes were observed and recorded. The significant elevation of the ST segment in ECG presented successful establishment of acute myocardial infarction model. The wound was sutured, followed by using penicillin to prevent the infection. After 4 weeks from surgery, the cardiac functions were detected using color echocardiography. The ejection fraction less than $45 \%$ was considered as a sign of CHF. The rats with death or failure to meet CHF criteria were excluded. The sham-operated group received the surgery the same with other 4 groups, excepting the coronary artery ligation.

\section{Detection of serum brain natriuretic peptide and interleukin 6 levels of rats}

At the end of treatment, $10 \mathrm{ml}$ of femoral vein blood was taken from the rats. The serum brain natriuretic peptide (BNP) and interleukin 6 (IL-6) levels were determined using radioimmunoassay method. The procedures were in accordance to the instructions of kits (Shanghai Sangon Biological Engineering Technology And Service Co., Ltd., Shanghai, China).

\section{Detection of cardiac function of rats}

After anaesthetizing using $10 \%$ chloral hydrate, the rats were fixed in supine position. The right carotid artery was separated. The ventricular catheter was inserted into the left ventricle through the right carotid artery, and was connected to the biological signal recorder to determine the left ventricular systolic pressure (LVSP), left ventricular end diastolic pressure (LVEDP) and maximum left ventricular systolic/diastolic rate $\left( \pm \mathrm{d} p / \mathrm{d} t_{\text {max }}\right)$.

\section{Determination of protein expressions related to cardiomyocyte apoptosis and ventricular remodelingregulation}

The rats were executed, and the heart of was taken. The myocardial tissue was homogenized, and the protein was extracted. The expressions levels of cardiomyocyte apoptosis related protein Caspase- 3 and Caspase-9, and ventricular remodelingregulation related protein MMP-1 and TIMP-1 in myocardial tissue were determined using western blot assays (Liu, Zhang, Li, 2011; Jin et al., 2014). The procedures were in accordance to the instructions of 
Protective effects of salidroside on chronic heart failure in rats and the underlying mechanisms

TABLE I - Effects of salidroside on cardiac function of rats

\begin{tabular}{|c|c|c|c|c|}
\hline Group & $\operatorname{LVSP}(\mathrm{kPa})$ & LVEDP (kPa) & $+\mathrm{d} p / \mathrm{d} t_{\max }(\mathrm{kPa} / \mathrm{s})$ & $-\mathrm{d} p / \mathrm{d} t_{\max }(\mathrm{kPa} / \mathrm{s})$ \\
\hline Sham-operated & $141.8 \pm 23.45$ & $0.08 \pm 0.03$ & $821.46 \pm 100.72$ & $765.21 \pm 121.73$ \\
\hline Model & $72.11 \pm 10.23^{*}$ & $0.62 \pm 0.21^{*}$ & $321.05 \pm 91.94^{*}$ & $267.33 \pm 44.92^{*}$ \\
\hline Low-dose salidroside & $80.91 \pm 21.02^{*}$ & $0.55 \pm 0.15^{*}$ & $332.19 \pm 73.04^{*}$ & $198.04 \pm 35.03^{* \#}$ \\
\hline Middle-dose salidroside & $111.27 \pm 12.49^{* \# \mathscr{E}}$ & $0.18 \pm 0.07^{* * \&}$ & $682.01 \pm 118.29^{* * \&}$ & $615.03 \pm 178.03^{* * \&}$ \\
\hline High-dose salidroside & $127.81 \pm 18.56^{\# \& \%}$ & $0.11 \pm 0.05^{\# \& \%}$ & $762.47 \pm 120.21^{\# \&}$ & $661.04 \pm 156.22^{\# \&}$ \\
\hline
\end{tabular}

${ }^{*} \mathrm{P}<0.05$ compared with sham-operated group; ${ }^{*} \mathrm{P}<0.05$ compared with model group; ${ }^{\&} \mathrm{P}<0.05$ compared with low-dose salidroside group; ${ }^{\%} \mathrm{P}<0.05$ compared with middle-dose salidroside group. LVSP, left ventricular systolic pressure; LVEDP, left ventricular end diastolic pressure; $\mathrm{d} p / \mathrm{d} t_{\max }$, maximum left ventricular systolic/diastolic rate.

kits (Beijing Zhongshan Golden Bridge Biotechnology Co., Ltd., Beijing, China).

\section{Statistical analysis}

The data were presented as mean \pm standard deviation, and were analyzed using SPSS 20.0 software (SPSS Inc., Chicago, IL, USA). Comparisons among different groups were performed using the single-factor analysis of variance test with $\mathrm{SNK}-\mathrm{q}$ test. $\mathrm{P}<0.05$ presented statistically significant.

\section{RESULTS}

\section{General situation and death of rats}

After establishment of CHF model, the rats in model group presented nose and lip cyanosis, pallor of extremities, rough color and yellowing, abdominal hair shedding, lags in response, reduced feed consumption, slow action and other CHF symptoms, which were gradually aggravated with time prolonging. During the treatment duration, the CHF symptoms of rats in salidroside groups were gradually alleviated, especially in high-dose salidroside group. At the end of treatment, the CHF symptoms of rats in high-dose salidroside group were obviously alleviated, with good blood flow, white and lustre hair, sensitive response, and basically normal food taking and activities. In model group there were 6 rats with death, with mortality rate of $30 \%$. No rat died in each other group.

\section{Salidroside improving the cardiac function of CHF rats}

As shown in Table I, compared with sham-operated group, in model group the LVEDP was significantly increased $(\mathrm{P}<0.05)$, and the LVSP, $+\mathrm{d} p / \mathrm{d} t_{\max }$ and $-\mathrm{d} p /$ $\mathrm{d} t_{\max }$ were significantly decreased, respectively $(\mathrm{P}<0.05)$.
Compared with model group, the LVSP and $+\mathrm{d} p / \mathrm{d} t_{\max }$ in middle- and high-dose salidroside groups, and the $-\mathrm{d} p /$ $\mathrm{d} t_{\max }$ in 3 salidroside groups were significantly increased, respectively $(\mathrm{P}<0.05)$. The LVEDP in middle- and highdose salidroside groups was significantly decreased, respectively $(\mathrm{P}<0.05)$.

\section{Salidroside decreasing the serum BNP and IL-6 levels in CHF rats}

Compared with sham-operated group, the serum BNP and IL-6 levels in model group were significantly increased, respectively $(\mathrm{P}<0.05)$. Compared with model group, the serum $\mathrm{BNP}$ level in high-dose salidroside group was significantly decreased $(\mathrm{P}<0.05)$, and serum IL-6 levels in middle- and high-dose salidroside groups were significantly decreased, respectively $(\mathrm{P}<0.05)$ (Table II).

TABLE II - Effects of salidroside on serum BNP and IL-6 levels in rats

\begin{tabular}{lcc}
\hline Group & BNP $\left(\times \mathbf{1 0}^{\mathbf{9}} \mathbf{g} / \mathbf{L}\right)$ & IL-6 $((\mathbf{p g} / \mathbf{m L})$ \\
\hline Sham-operated & $444.93 \pm 103.52$ & $24.32 \pm 2.94$ \\
Model & $598.23 \pm 126.92^{*}$ & $33.52 \pm 3.87^{*}$ \\
Low-dose salidroside & $553.01 \pm 138.27^{*}$ & $32.72 \pm 2.96^{*}$ \\
Middle-dose salidroside & $497.34 \pm 128.03^{\&}$ & $28.04 \pm 3.21^{* \# \&}$ \\
High-dose salidroside & $483.67 \pm 78.03^{\# \&}$ & $25.16 \pm 2.64^{\# \&}$ \\
\hline
\end{tabular}

${ }^{*} \mathrm{P}<0.05$ compared with sham-operated group; ${ }^{*} \mathrm{P}<0.05$ compared with model group; ${ }^{\mathrm{P}}<0.05$ compared with lowdose salidroside group. BNP, brain natriuretic peptide; IL-6, interleukin 6.

\section{Salidroside regulating myocardial Caspase-3 and Caspase-9 protein expressions in CHF rats}

As shown in Figure 1, compared with sham-operated group, the expression levels of myocardial Caspase-3 
and Caspase-9 protein in model group were significantly increased, respectively $(\mathrm{P}<0.05)$. Compared with model group, the expression level of Caspase- 3 protein in highdose salidroside group was significantly decreased (P $<0.05$ ), and the expression levels of Caspase-9 protein in middle- and high-dose salidroside groups were significantly decreased, respectively $(\mathrm{P}<0.05)$.

\section{Salidroside regulating myocardial MMP-1 and TIMP-1 protein expressions in CHF rats}

Compared with sham-operated group, in model group the expression level of myocardial MMP-1 protein

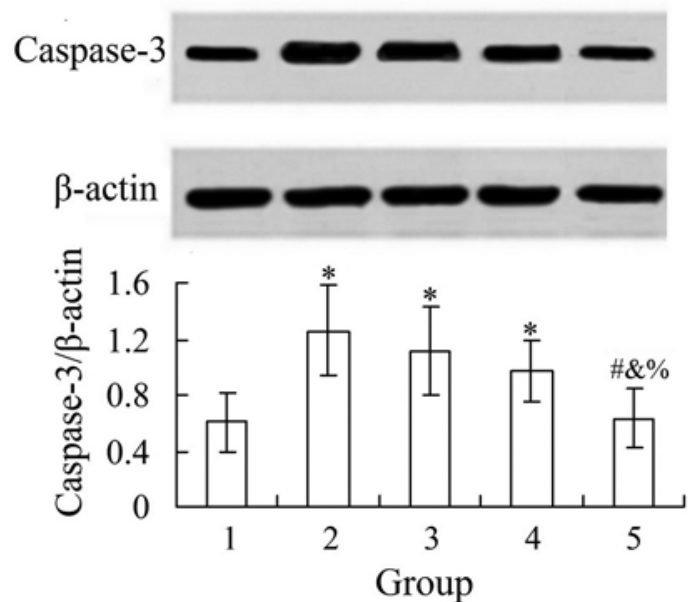

was significantly increased $(\mathrm{P}<0.05)$, and the expression level of TIMP-1 protein was significantly decreased $(\mathrm{P}<$ $0.05)$. Compared with model group, the expression levels of MMP-1 protein in middle- and high-dose salidroside groups were significantly decreased, respectively ( $\mathrm{P}$ $<0.05$ ), and the expression levels of TIMP-1 protein in 3 salidroside groups were significantly increased, respectively $(\mathrm{P}<0.05)$ (Figure 2$)$.

\section{DISCUSSION}

Salidroside has the anti-hypoxia, anti-myocardial ischemia, anti-myocardial apoptosis, anti-oxidative stress,
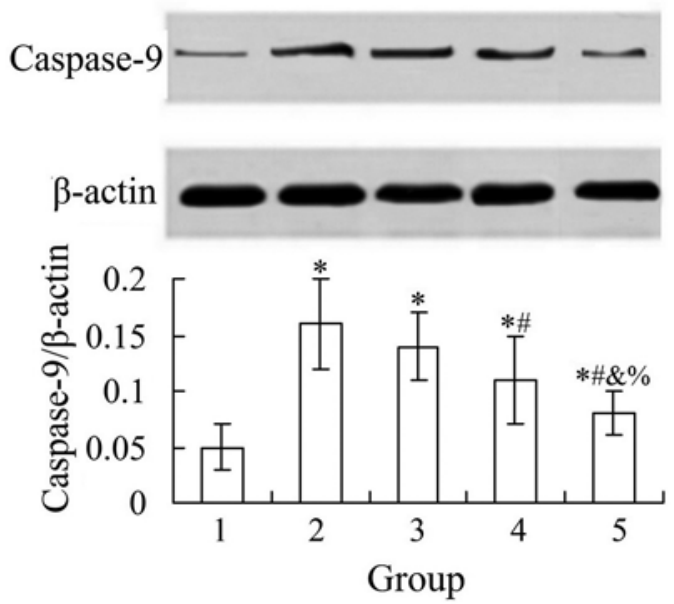

FIGURE 1 - Effects of salidroside on myocardial Caspase-3 and Caspase- 9 protein expressions in rats. ${ }^{*} \mathrm{P}<0.05$ compared with sham-operated group; ${ }^{\mathrm{P}}<0.05$ compared with model group; ${ }^{\&} \mathrm{P}<0.05$ compared with low-dose salidroside group; $\% \mathrm{P}<0.05$ compared with middle-dose salidroside group. 1: Sham-operated group; 2: Model group; 3: low-dose salidroside group; 4: middledose salidroside group; 5 : high-dose salidroside group. Caspase-3, cysteinyl aspartate-specific proteinase-3; Caspase-9, cysteinyl aspartate-specific proteinase-9.
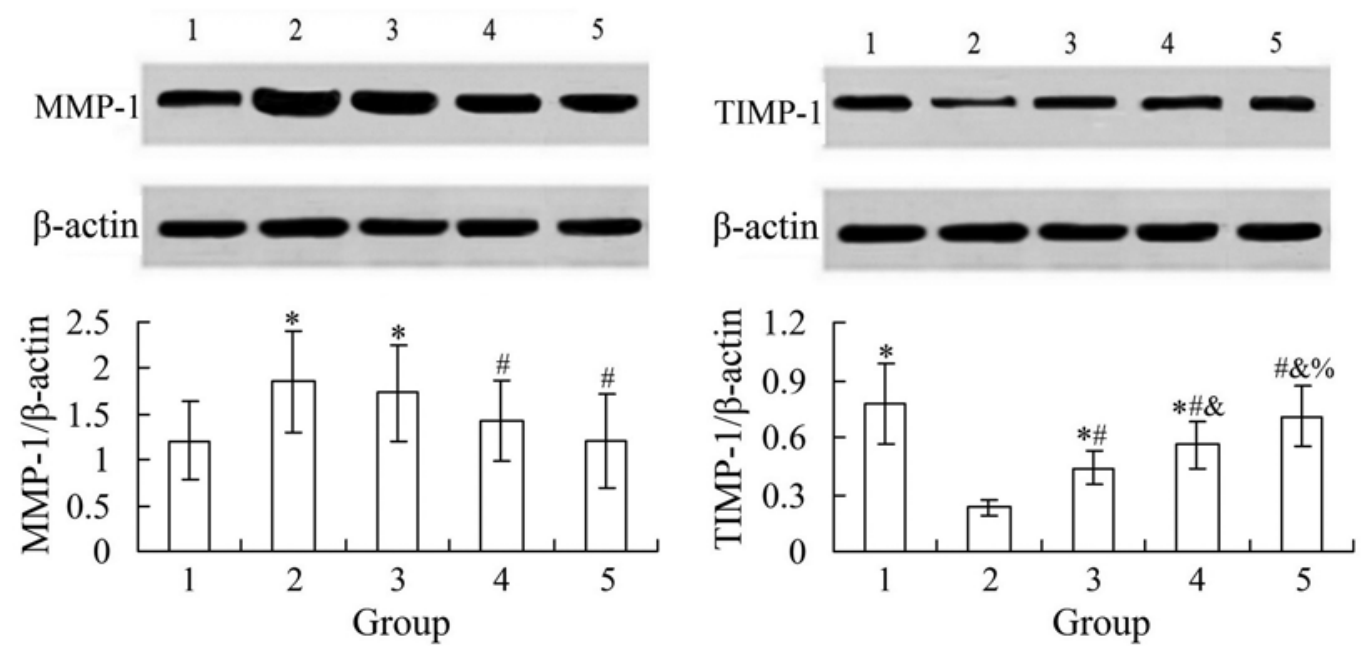

FIGURE 2 - Effects of salidroside on myocardial MMP-1 and TIMP-1 protein expressions in rats. * $\mathrm{P}<0.05$ compared with shamoperated group; ${ }^{*} \mathrm{P}<0.05$ compared with model group; ${ }^{\&} \mathrm{P}<0.05$ compared with low-dose salidroside group; $\% \mathrm{P}<0.05$ compared with middle-dose salidroside group. 1: Sham-operated group; 2: Model group; 3: low-dose salidroside group; 4: middle-dose salidroside group; 5: high-dose salidroside group. MMP-1, matrix metalloproteinase-1; TIMP-1, tissue inhibitor of metalloproteinase-1. 
anti-nerve cell damage, immunity regulation and sleep improvement effects, and is widely used in the prevention and treatment of hypertension, stroke, neurasthenia and other diseases (Huang et al., 2015). Studies (Zheng et al., 2017; Sun et al., 2018) have confirmed that, salidroside can inhibit the apoptosis of vascular endothelial cells, reduce the expression of vascular endothelial growth factor in atherosclerotic plaques, and inhibit the angiogenesis, thereby blocking the formation of atherosclerosis. In addition, salidroside can block the mitochondrial pathway to reduce the apoptosis of ischemic cardiomyocytes, and protect the heart from reperfusion injury (Wu et al., 2009). However, whether salidroside plays a role in CHF and its specific mechanisms remain to be further studied. In this study, the CHF of rat model was established, and the effects of salidroside on CHF and the underlying mechanisms were investigated. Results showed that, after 4 weeks of treatment, the rats in model group presented obvious CHF symptoms. Compared with model group, the CHF symptoms in salidroside groups were obviously alleviated. The mortality rate of rats in salidroside groups was significantly lower than model group. In addition, the heart function indexes in model group were obviously deteriorated. Compared with model group, in salidroside group the heart function indexes of rats were significantly improved. This indicates that, salidroside has obvious protective effects on $\mathrm{CHF}$ in rats.

BNP is a polypeptide hormone composed of 32 amino acids. It is mainly synthesized and secreted by the ventricle. When the ventricular volume load and wall tension change, the amount of BNP produced by ventricle increases. Therefore, the concentration of BNP in blood can directly reflect the function of ventricle (Kallistratos et al., 2008). It is reported that, the plasma concentration of BNP in CHF patients is higher than that in normal people, and it is positively related to the severity of heart failure (Mowla, Bustami, 2006). IL-6 is a cytokine secreted by activated macrophages, and it has a wide range of biological effects in the body. On the one hand, IL-6 has the antiviral, antibacterial and antiparasitic function (Fostok et al., 2009). On the other hand, IL-6 can cause serious damage to the body by participating in some serious pathophysiological states such as septic shock and malignant fluid (Martin et al., 1997). Previous study (Tsutamoto et al., 1998) has shown that, IL-6 plays wide pathophysiological roles in the development of heart failure. The IL- 6 concentration in heart failure patients is significantly higher than that of the normal control group, and the IL-6 level is positively correlated with cardiac function level, that is, the more serious the heart failure is, the higher the IL-6 concentration is. Results of this study showed that, compared with sham-operated group, the serum BNP and IL-6 levels in model group were significantly increased, respectively. Compared with model group, the serum BNP level in high-dose salidroside group was significantly decreased, and serum IL-6 levels in middle- and high-dose salidroside groups were significantly decreased, respectively. This indicates that, salidroside can alleviate CHF in rats by regulating the level of neuroendocrine cytokines.

Apoptosis is a process of automatic life ending which is controlled by the internal genetic mechanism under certain physiological or pathological conditions. Apoptosis is involved in the ventricular remodeling, and plays an important role in the evolution of heart failure (Palojoki et al., 2001). The occurrence of apoptosis is mediated by the exogenous and endogenous pathways which exert the functions by activating Caspases. In Caspases family, Caspase- 3 is the most important apoptosis protease in downstream of apoptotic cascade reaction. Caspase-9 is in the upstream of apoptotic cascade reaction. Caspase-9 is activated by the apoptosome formed under the promotion of various apoptotic factors, and acts with Caspase-3, thus executing the apoptotic program (Cowan, Roskams, 2004). In this study, compared with sham-operated group, the expression levels of Caspase-3 and Caspase-9 protein in myocardial tissue of rats in model group were significantly increased, respectively. Compared with model group, the expression level of Caspase-3 protein in high-dose salidroside group was significantly decreased, and the expression levels of Caspase- 9 protein in middle- and high-dose salidroside groups were significantly decreased, respectively. This indicates that, Caspase- 3 and Caspase- 9 are involved in the $\mathrm{CHF}$ in rats, and salidroside can down-regulate the myocardial Caspase- 3 and Caspase- 9 protein expressions in CHF rats, thus exerting the protection effect.

Ventricular remodeling is the basic pathological link of occurrence and development of CHF, and the change of extracellular matrix is an important part of ventricular remodeling. Matrix metalloproteinases are the most important enzymes in the degradation of extracellular matrix of myocardium, and are the most important driving force for ventricular remodeling (Sakata et al., 2004). It has been shown that, the activation of MMP-1 plays an important role in cardiac remodeling induced by extracellular matrix remodeling. TIMP-1 is a specific inhibitor of MMP-1. The balance between MMP-1 and TIMP-1 is important for maintaining the stability of cardiac collagen metabolism (Picard et al., 2006). Results of this study showed that, compared with sham-operated group, in model group the expression level of MMP-1 
protein in myocardial tissue of rats was significantly increased, and the expression level of TIMP-1 protein was significantly decreased. Compared with model group, the expression levels of MMP-1 protein in middle- and high-dose salidroside groups were significantly decreased, respectively, and the expression levels of TIMP-1 protein in 3 salidroside groups were significantly increased, respectively. This indicates that, MMP-1 and TIMP-1 are also involved in CHF in rats, and the protection effect of salidroside is related to its regulation of MMP-1 and TIMP-1 protein expressions in myocardial tissue.

\section{CONCLUSION}

Salidroside has obvious protective effects on CHF in rats. The mechanism may be related to its regulation of cardiomyocyte apoptosis protein Caspase-3 and Caspase- 9 expressions and ventricular remodelingregulation protein MMP-1 and TIMP-1 expressions in myocardial tissue. This study has provided an experimental basis for the application of salidroside to clinical treatment of CHF. This study still has some limitations. The dose-effect relationship of salidroside on CHF is not very clear, and more salidroside doses should be adopted in next studies. In addition, whether there are correlations among these proteins and whether there are other mechanisms of salidroside in alleviating CHF should be further investigated.

\section{REFERENCES}

Al-Mohammad A, Mant J. Republished technology and guidelines: the diagnosis and management of chronic heart failure: review following the publication of the NICE guidelines. Postgrad Med J. 2011;87(1034):841-846.

Balakumar P, Singh M. The possible role of caspase-3 in pathological and physiological cardiac hypertrophy in rats. Basic Clin Pharmacol Toxicol. 2006;99(6):418-424.

Cleland JG, Zhang J, Pellicori P, Dicken B, Dierckx R, Shoaib A, Wong K, Rigby A, Goode K, Clark AL. Prevalence and outcomes of anemia and hematinic deficiencies in patients with chronic heart failure. JAMA Cardiol. 2016;1(5):539-547.

Cowan CM, Roskams AJ. Caspase-3 and caspase-9 mediate developmental apoptosis in the mouse olfactory system. J Comp Neurol. 2004;474(1):136-148.
Distefano G, Sciacca P. Molecular pathogenesis of myocardial remodeling and new potential therapeutic targets in chronic heart failure. Ital J Pediatr. 2012;38(1):41.

Fostok SF, Ezzeddine RA, Homaidan FR, Al-Saghir JA, Salloum RG, Saliba NA, Talhouk RS. Interleukin-6 and Cyclooxygenase- 2 downregulation by fatty-acid fractions of Ranunculus constantinopolitanus. BMC Complement Altern Med. 2009;9:44.

Han XJ, Guo N, Zhu MX, Tao Y. Research progress in pharmacological activities and related mechanism of salidroside. Chin J Biochem Pharm, 2015;35(1):171-175.

Huang X, Zou L, Yu X, Chen M, Guo R, Cai H, Yao D, Xu X, Chen Y, Ding C, Cai X, Wang L. Salidroside attenuates chronic hypoxia-induced pulmonary hypertension via adenosine A2a receptor related mitochondria-dependent apoptosis pathway. J Mol Cell Cardiol. 2015;82:153-166.

Jin J, Ma L, Zou G, Liu L. Impacts of Shenqi Yixin formula on the protein expression of Caspase 3 and Caspase 9 in myocardial tissue in the rats of chronic heart failure. World J Integ Trad West Med. 2014;9(2):151-155 (in Chinese).

Kallistratos MS, Dritsas A, Laoutaris ID, Cokkinos DV. Incremental value of $\mathrm{N}$-terminal pro-brain natriuretic peptide over left ventricle ejection fraction and aerobic capacity for estimating prognosis in heart failure patients. J Heart Lung Transplant. 2008;27(11):1251-1256.

Liu X, Zhang Y, Li H. Effect of traditional Chinese medicine with benefiting Qi and activating blood circulation on MMP-1 and TIMP-1 expression in rats with chronic heart failure. Tianjin J Tradit Chin Med. 2011;28(6):503-505 (in Chinese).

Martin C, Boisson C, Haccoun M, Thomachot L, Mege JL. Patterns of cytokine evolution (tumor necrosis factor-alpha and interleukin-6) after septic shock, hemorrhagic shock, and severe trauma. Crit Care Med. 1997;25(11):1813-1819.

Mowla MM, Bustami BB. Brain natriuretic peptide in heart failure and beyond. Saudi Med J. 2006;27(10):1457-1461.

Palojoki E, Saraste A, Eriksson A, Pulkki K, Kallajoki M, Voipio-Pulkki LM, Tikkanen I. Cardiomyocyte apoptosis and ventricular remodeling after myocardial infarction in rats. Am J Physiol Heart Circ Physiol. 2001;280(6):H2726-2731. 
Picard F, Brehm M, Fassbach M, Pelzer B, Scheuring S, Küry P, Strauer BE, Schwartzkopff B. Increased cardiac mRNA expression of matrix metalloproteinase-1 (MMP-1) and its inhibitor (TIMP-1) in DCM patients. Clin Res Cardiol. 2006;95(5):261-269.

Sakata Y, Yamamoto K, Mano T, Nishikawa N, Yoshida J, Hori M, Miwa T, Masuyama T. Activation of matrix metalloproteinases precedes left ventricular remodeling in hypertensive heart failure rats: its inhibition as a primary effect of Angiotensin-converting enzyme inhibitor. Circulation. 2004;109(17):2143-2149.

Su Q, Li L, Liu YC, Zhou Y, Lu YG, Wen WM. Effect of metoprolol on myocardial apoptosis and caspase-9 activation after coronary microembolization in rats. Exp Clin Cardiol. 2013;18(2):161-165.

Sun L, Dou F, Chen J, Chi H, Xing S, Liu T, Sun S, Chen C. Salidroside slows the progression of EA.hy926 cell senescence by regulating the cell cycle in an atherosclerosis model. Mol Med Rep. 2018;17(1):257-263.

Tsutamoto T, Hisanaga T, Wada A, Maeda K, Ohnishi M, Fukai D, Mabuchi N, Sawaki M, Kinoshita M. Interleukin-6 spillover in the peripheral circulation increases with the severity of heart failure, and the high plasma level of interleukin-6 is an important prognostic predictor in patients with congestive heart failure. $\mathrm{J}$ Am Coll Cardiol. 1998;31(2):391-398.

Wu T, Zhou H, Jin Z, Bi S, Yang X, Yi D, Liu W. Cardioprotection of salidroside from ischemia/reperfusion injury by increasing $\mathrm{N}$-acetylglucosamine linkage to cellular proteins. Eur J Pharmacol. 2009;613(1-3):93-99.
Yin H, Zhang J, Lin H, Qiao Y, Wang R, Lu H, Liang S. Effect of traditional Chinese medicine Shu-mai-tang on angiogenesis, arteriogenesis and cardiac function in rats with myocardial ischemia. Phytother Res. 2009;23(1):92-98.

Yin HQ, Wang B, Zhang JD, Lin HQ, Qiao Y, Wang R, Liu FY. Effect of traditional Chinese medicine Shu-Mai-Tang on attenuating TNFalpha-induced myocardial fibrosis in myocardial ischemia rats. J Ethnopharmacol. 2008;118(1):133139.

Zhang J, Liu A, Hou R, Zhang J, Jia X, Jiang W, Chen J. Salidroside protects cardiomyocyte against hypoxia-induced death: a HIF-1alpha-activated and VEGF-mediated pathway. Eur J Pharmacol. 2009;607(1-3):6-14.

Zhang J, Zhen YF, Pu-Bu-Ci-Ren, Song LG, Kong WN, Shao TM, Li X, Chai XQ. Salidroside attenuates beta amyloidinduced cognitive deficits via modulating oxidative stress and inflammatory mediators in rat hippocampus. Behav Brain Res. 2013;244:70-81.

Zheng XT, Wu ZH, Wei Y, Dai JJ, Yu GF, Yuan F, Ye LC. Induction of autophagy by salidroside through the AMPKmTOR pathway protects vascular endothelial cells from oxidative stress-induced apoptosis. Mol Cell Biochem. 2017;425(1-2):125-138.

Received for publication on $21^{\text {st }}$ March 2018 Accepted for publication on $16^{\text {th }}$ April 2018 